\title{
Quantitative Phase Analysis of Martian Meteorite Alteration
}

\author{
E.P. Vicenzi* and M. Velbel** \\ * $\quad$ Museum Conservation Institute, Smithsonian Institution, Suitland, MD 20746 \\ ** Department of Geological Sciences, Michigan State University, East Lansing, MI 48824
}

Information regarding aqueous processes on Mars is typically derived from spacecraft-mounted remote sensing instrumentation. An alternative source of such data can be obtained from direct microscopic examination of low temperature precipitates in Martian meteorites, provided that one can demonstrate that the aqueous deposition took place preterrestrially, or before the meteorite was delivered to our planet(1). Because most meteorites have a significant terrestrial age, the time elapsed between impact and collection, one must also consider subsequent terrestrial low temperature chemical modification. To evaluate these two potential periods of alteration the fusion crust, a partly molten skin formed by frictional heating during entry into Earth's atmosphere, is a useful region of a meteorite to study since it provides a certain terrestrial time-stamp. Martian meteorite MIL03346 (from the Miller Range ice fields of Antarctica) was studied using hyperspectral X-ray microanalysis as part of a effort to understand the water-rock interaction history of the meteorite (Fig. 1 A, B) [2].

An FEI NanoSEM600 microscope equipped with a Thermo Scientific NSS302 X-ray system was used to collect electron-excited X-ray data cubes at 7 and $13 \mathrm{KeV}$ accelerating energies. Analysis was performed in two steps: (1) reduction of dimensionality of the hyperspectral data set by multivariate statistical analysis (MVS) using the solution obtained when spatial contrast among the pure components is maximized (Fig. 2 A-E) [3,4], and (2) 16 bit MVS component image output was used as input for further processing by the NSS XPhase image correlation program to segment the analysis region into binary chemical phase masks with their associated derived spectra (Fig. 2 F-J).

The prominent veinlet shown in Fig. 1A cross-cuts, and therefore postdates, formation of the fusion crust. Hence, the materials in this veinlet were deposited in Antarctica. Interestingly, the terrestrial assemblage is a multiphase mixture including, Ca-sulfate (anhydrite), a cryptocrystalline Al-bearing Si-rich phase, and K-Fe sulfate (jarosite) mixed with the Al-bearing silicate at scale less than the excited analytical volume. Additionally, a $\mathrm{Mg}-\mathrm{Ca}-\mathrm{Fe}$ silicate phase may represent the sum of a preterrestrial veinlet altered by fusion crust melt, and is spectrally indistinguishable from phasemixed pixels of the terrestrial veinlet and the $\mathrm{Ca}-\mathrm{Mg}$ silicate (Ca pyroxene) phase which represents the high temperature host crystal. The complexity of the phase microstructure (Fig. 1C) suggests multiple episodes of terrestrial fluid-meteorite interaction.

Complex mixtures such as those described here have previously been improperly analyzed by overscanning the electron beam (or defocusing the beam) and summing the X-rays from all phases into a single spectrum. The reduction of such admixed raw X-ray data of distinct phases cannot yield an accurate corrected microchemical composition. Segmenting phases, and summing pixels for each phase, must be performed before applying background and matrix corrections to obtain a meaningful compositions within chemically complex microstructures. 
References

[1] A.H. Treiman, Meteoritics and Planetary Science 28, (1993) 86.

[2] M.A. Velbel et al., Lunar and Planetary Science XLI, (2010) \#2223 CD

[3] M.R. Keenan and P.G. Kotula, Microscopy and Microanalysis (2004), 10(Suppl

2):874-875CD

[4] M.R. Keenan, Surface and Interface Analysis, 41, (2009) 79-87.
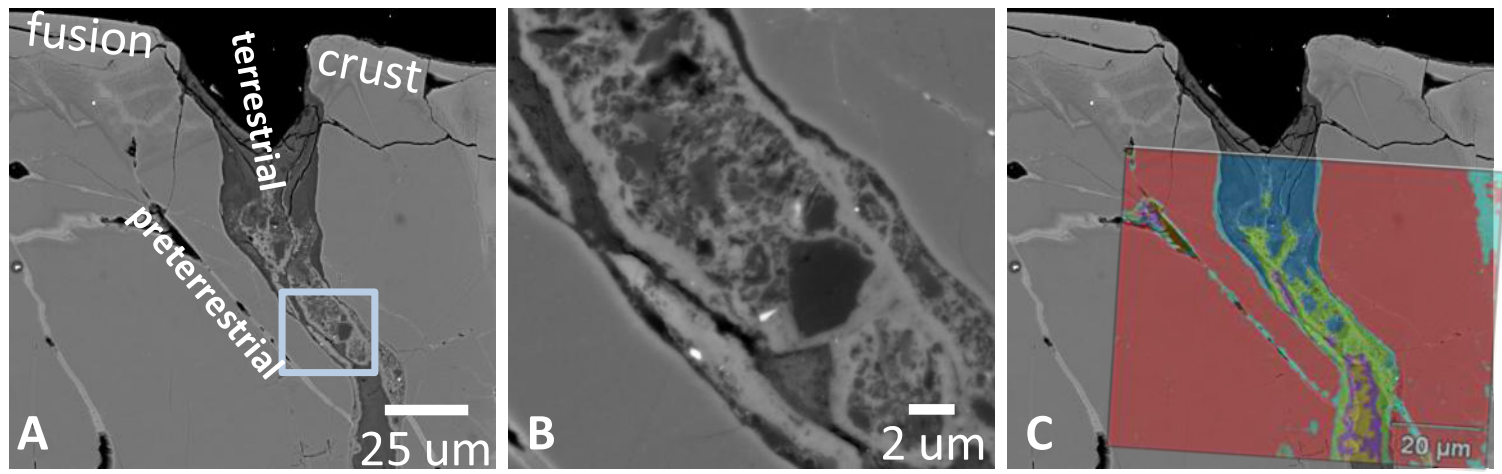

FIG 1. A) Backscattered electron image (BSE) of the MIL03346 meteorite fusion crust in the vicinity of alteration veinlets. B) Higher resolution BSE image of a terrestrial veinlet displaying textural complexity among precipitate pulses at the submicrometer lengthscale.

C) BSE image A with false color chemical phase composite overlay.

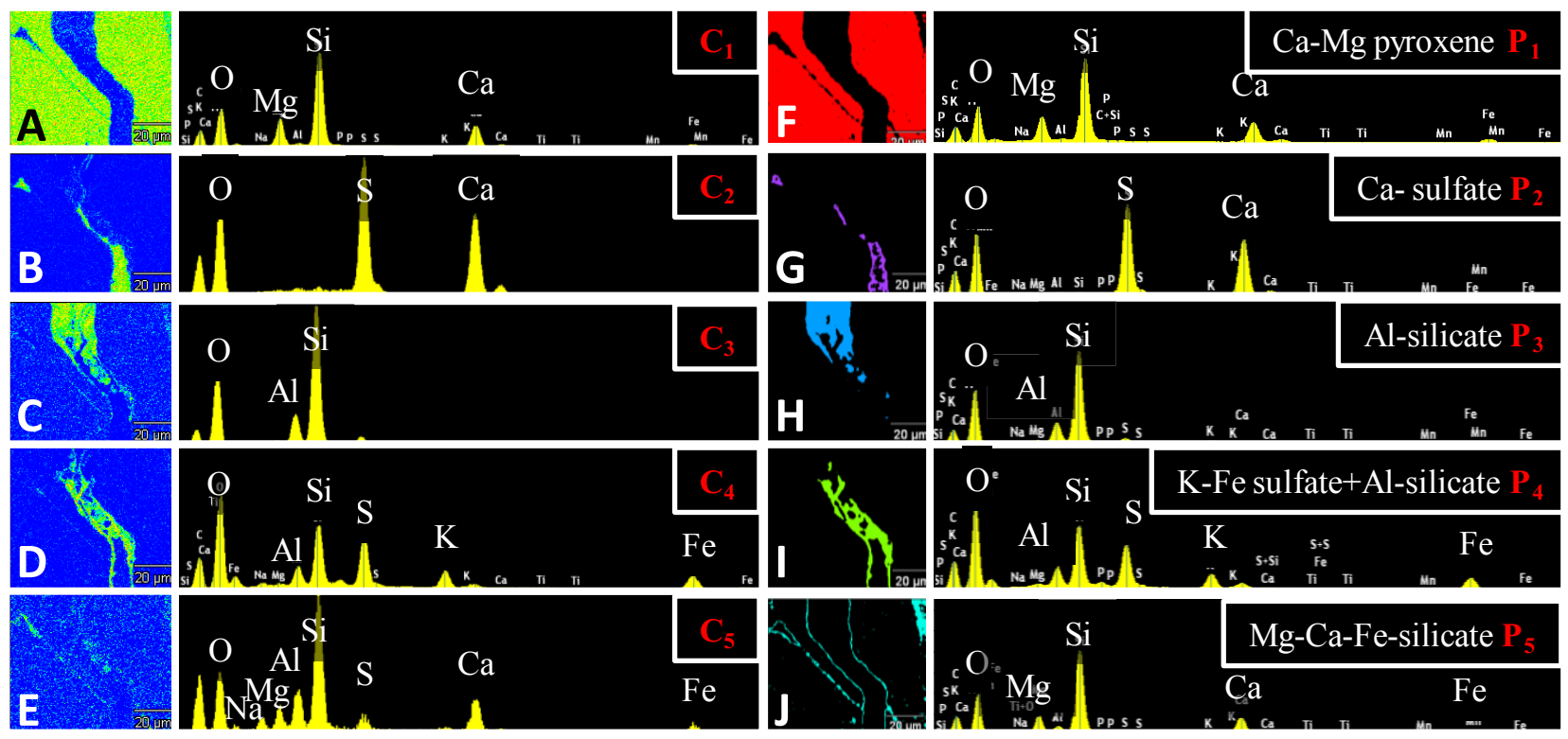

FIG 2. A-E) Spatial domain simplicity solution for an X-ray hyperspectral data cube collected in the region shown in $1 \mathrm{C}$ showing paired intensity maps for multivariate statistical pure-components and their associated computed spectra. F-J) Binary image masks depicting chemical phases for the high temperature host crystal $(\mathrm{F})$, low temperature alteration precipitates $(\mathrm{G}-\mathrm{J})$, and their associated spectra derived directly from the data cube. 